Internist 2015 · 56:1231-1232

DOI 10.1007/s00108-015-3709-9

Online publiziert: 21. Oktober 2015

(c) Springer-Verlag Berlin Heidelberg 2015

\section{B. Salzberger ${ }^{1} \cdot$ T. Welte $^{2}$}

${ }^{1}$ Stabsstelle Infektiologie, Universitätsklinikum Regensburg, Regensburg

${ }^{2}$ Klinik für Pneumologie, Medizinische Hochschule Hannover, Hannover

\title{
Multiresistente Erreger
}

Antibiotika sind wunderbare Medikamente. Sie können bei schweren Infektionen lebensrettend sein und verbessern die Prognose komplizierter Operationen. Außerdem ist ihre Sicherheit bemerkenswert, bei vielen Substanzen sind schwere Nebenwirkungen und auch Interaktionen sehr selten. Die Kosten spiegeln den Wert nicht wider, bis auf Ausnahmen unterliegen die wichtigen Substanzen längst nicht mehr dem Patentschutz. Eigentlich goldene Zeiten für die Behandlung bakterieller Infektionen ...

Zyniker schwärmen, wie schön Schulen ohne Schüler und Krankenhäuser ohne Patienten sein könnten; nur Zyniker können die derzeitigen Therapieaussichten bei bakteriellen Infektionen für gut halten - ohne Bakterien. Bemerkenswert viele Spezies haben sich in den letzten Jahrzehnten gut gegen den Angriff durch Antibiotika gewappnet, am besten jene, die in engem Kontakt zum Menschen leben, z. B. Staphylococcus aureus und viele gramnegative Enterobakterien. Unser Fokus lag jahrelang auf der ersteren Spezies - MRSA war als Schlagwort auch in der Laienpresse bald bekannt. Die Entwicklung bei den gramnegativen Erregern hat uns dagegen in ihrem Ausmaß und ihrer Dynamik überrascht [1]. Die Mechanismen sind nicht neu: Resistenzgene waren bei Bakterien lange vor der Anwendung von Antibiotika vorhanden. Neu sind aber die rasche Zunahme der Häufigkeit dieser Resistenzen und ihre weltweite Ausbreitung. Dem steht ein jahrelanger Stillstand in der Forschung zu neuen Antiinfektiva gegenüber. Diese beiden Trends charakterisieren die augenblickliche gefährliche Situation, bereits jetzt treten Infektionen mit kaum behandel- baren Bakterien auf, und das gerade in der Hochleistungsmedizin. Insbesondere hier nämlich, bei Patienten mit schwerer Immunsuppression oder Implantaten, sind die therapeutischen Probleme und Herausforderungen durch häufige und schwere Infektionen am größten.

\section{》) Resistenzgene existierten lange vor der Anwendung von Antibiotika}

Nicht nur die zuvor genannten Erreger machen uns Sorgen. Auch bei der Tuberkulose ist die Resistenzentwicklung dramatisch, Brennpunkte sind weiterhin Osteuropa, Zentralasien und das südliche Afrika. Diese Erkrankung wird durch die zunehmende Migration auch bei uns häufiger werden, mit und ohne Resistenzen. Die vielfältigen speziellen Aspekte dieses Themas werden deshalb in einem der nächsten Schwerpunkte gesondert dargestellt.

Der Weg aus der Krise erfordert große Anstrengungen. Zunächst müssen wir die Ursachen kühl und klar analysieren, sonst laufen wir Gefahr, erneut in dieselbe Sackgasse zu geraten. Drei Aspekte sind hier zentral: die Mechanismen von Resistenzentwicklung und -ausbreitung, der adäquate Einsatz von Antibiotika und die Entwicklung neuer antiinfektiver Therapien (Antibiotika und andere Ansätze). Forschung und Innovationen in all diesen Bereichen sind dringend notwendig. In der Entstehung und Ausbreitung von Resistenzen spielt der Einsatz von Antibiotika sowohl bei Menschen als auch bei Tieren sicher eine Rolle. Resistenzen sind meist zuerst im Gefolge der Einführung neuer Antibiotika beobachtet worden und dann vorrangig bei Patienten mit häufiger Antibiotikabehandlung. Aber Resistenzgene sind viel älter als Antibiotika, sie gehören quasi zum Abwehrsystem der Bakterien. Viel wichtiger ist hier die Frage nach den molekularen Mechanismen. Die Entstehung neuer Resistenzen kann durch die evolutionäre Entwicklung von Resistenzgenen, aber auch durch den horizontalen Gentransfer zwischen Bakterien derselben oder einer unterschiedlichen Spezies angetrieben werden. Welche Potenziale für die evolutionäre Entwicklung von $\beta$-Laktamasen bestehen bzw. welche Signale einen Genaustausch auslösen ist noch weitestgehend unbekannt. Auch der Austausch zwischen verschiedenen Ökosystemen, Bakterien aus dem Erdreich, bei Nutz- und Wildtieren und beim Menschen ist noch viel zu lückenhaft untersucht, um hieraus Konsequenzen abzuleiten (s. Beitrag von Käsbohrer). Relativ neu und unvollständig sind unsere Erkenntnisse zur Azolresistenz, v. a. bei Schimmelpilzen. Ein großes Problem ist hier auch der Nachweis (s. Beitrag von Vehreschild).

Als Sofortmaßnahme wird zurecht allseits eine gute Hygiene im Krankenhaus gefordert. Hier muss gerade verhindert werden, dass bei medizinischen Maßnahmen resistente Bakterien von einem auf den nächsten Patienten übertragen werden. Vor allem bei den o. g. Risikopatienten ist die Gefahr, dass aus einer Kolonisation eine Infektion wird, besonders hoch. Aber auch in diesem Kontext müssen Maßnahmen auf Wirksamkeit geprüft werden, bevor in sie viel Arbeit und Geld investiert werden. Es ist noch nicht lange her, dass in Deutschland mehr Aufwand in die Desinfektion 
des Fußbodens als in die der Hände gesteckt wurde. Glücklicherweise haben sich die Präferenzen geändert. Ein Forschungsdefizit besteht in Deutschland allerdings noch immer, wie auch ein großes Personaldefizit (s. Beitrag von Lemmen). Für beide Probleme muss auch an innovative Lösungen gedacht werden, z. B. an den Einsatz pädagogisch geschulter Biologen oder biologisch geschulter Pädagogen als „Hygienetrainer“.

Die Entwicklung neuer antiinfektiver Therapien ist sowohl eine Sofort- als auch eine Langzeitmaßnahme. Eine Reihe von neuen Antiinfektiva befindet sich in der fortgeschrittenen klinischen Entwicklung. Am weitesten entfernt vom klinischen Einsatz sind Substanzen mit Wirksamkeit gegen die in Asien weit verbreiteten Metallo- $\beta$-Laktamasen. Eine weit in die Zukunft weisende Entwicklung ist die von Teixobactin, einer ganz neuen Substanz, deren Entwicklung erst durch innovative Kulturverfahren möglich wurde (s. Beitrag von Kern). Die Entdeckung und Charakterisierung bisher unbekannter oder nicht kultivierbarer Bakterien wird hier sicher noch möglich sein. Und vielleicht werden dabei auch Substanzen mit anderer, nichtantibiotischer Wirksamkeit entdeckt, die in der Therapie von Infektionen einsetzbar sind, z. B. zur Modulation von Pathogenitätsfaktoren oder Verhinderung eines horizontalen Gentransfers.

\section{》) Die Wege der klinischen Prüfung von Antiinfektiva müssen hinterfragt werden}

Zusätzlich zur Entwicklung neuer Antiinfektiva müssen auch Wege der klinischen Prüfung hinterfragt und ggf. neu gefunden werden. Die klinische Prüfung von Substanzen, die in kritischen Situationen lebensrettend sein können, kann nicht mit den gleichen Maßstäben bewertet werden wie die Prüfung von Blockbuster-Medikamenten. Die zuständigen öffentlichen Fördereinrichtungen und Behörden müssen nach neuen Wegen der Arzneimittelprüfung suchen, um auch Neuentwicklungen von Arzneimitteln zu ermöglichen, die vermutlich keine hohen Gewinne ab- werfen werden. Mit den InnovativeMedicine-Initiative-Ausschreibungen der Europäischen Union sind erste Projekte entstanden. Von einer konstruktiven und akzeptierten öffentlich-privaten Partnerschaft sind wir jedoch noch weit entfernt.

Der „richtige“, nämlich gezielte und sorgfältige Einsatz von Antibiotika ist ein unverzichtbarer Baustein, die Korrelation von Resistenzentwicklung und Antibiotikaeinsatz ist eindeutig. Antibiotika sind zwar billig, aber gesellschaftlich und damit volkswirtschaftlich ein wertvolles Gut. Ein ungezielter Einsatz bedingt Resistenzentwicklungen, vermindert die Lebensdauer von Antibiotika und verursacht hohe Kosten für früher notwendige Neuentwicklungen [2].

Aber die Änderung von eingefahrenen Gewohnheiten bei Ärzten, Tierärzten und auch Patienten wird viel Aufklärungsarbeit und Einsatz in Aus-, Weiter- und Fortbildung erfordern. Und ähnlich wie Hygienemaßnahmen werden Maßnahmen der Antibiotic Stewardship nicht immer freudig begrüßt, sondern auch als Eingriff in die therapeutische Freiheit aufgefasst werden und daher auf Widerstand stoßen. Auch hier ist die wissenschaftliche Evaluation der Maßnahmen notwendig, nur mit guter Evidenz kann die Überzeugung gelingen (s. Beitrag von de With). Um den Wert von Antibiotika, der aktuellen wie auch der kommenden, zu erhalten, ist sicher ein Umdenken notwendig: In der Humanmedizin benötigt ein Patient mit Virusinfekt der oberen Luftwege kein Antibiotikum. Auch der Einsatz von Antibiotika aus anderen Gründen - u. a. zur Erzielung besserer Mastergebnisse bei Nutztieren oder zur Förderung der Darmmotilität durch Erythromycin - muss unterbleiben. Am ehesten kann dies durch eine breite Konsensbildung und Koalition der Beteiligten erreicht werden.

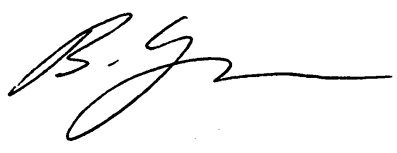

B. Salzberger

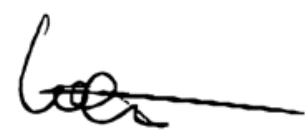

T. Welte

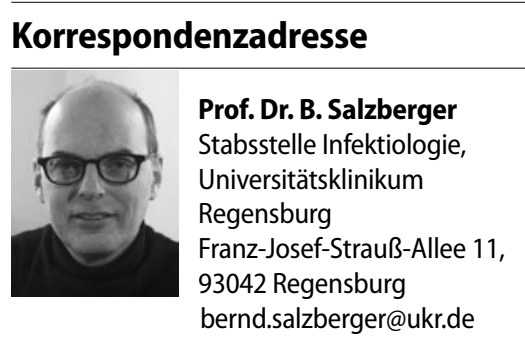

Interessenkonflikt. B. Salzberger und T. Welte geben an, dass kein Interessenkonflikt besteht.

\section{Literatur}

1. Nordmann P, Poirel L (2014) The difficult-to-control spread of carbapenemase producers among Enterobacteriaceae worldwide. Clin Microbiol Infect 20(9):821-830

2. Laxminarayan R (2014) Antibiotic effectiveness: balancing conservation against innovation. Science 345(6202):1299-1301 